\title{
Potential Utility of Adjunct Imaging with Wide-Field Optical Coherence Tomography for Gross and Microscopic Evaluation of Breast Specimens in Real-Time in the Operating Suite
}

\author{
Beth B. DuPree ${ }^{1}$ Michael J. Papez ${ }^{1}$. Elaina Pirruccello ${ }^{1}$ Audrey Hassenflug ${ }^{1}$
}

Received: 30 July 2021 / Accepted: 6 August 2021 / Published online: 26 November 2021

(C) The Author(s) 2021, corrected publication 2022

\begin{abstract}
One challenge in the surgical management of breast cancer is maximizing the preservation of healthy tissue while achieving acceptable negative margins. Tools capable of assessing disease-margin involvement intraoperatively and in real-time could provide clinically useful guidance regarding the adequacy of margin resection before the surgery is over. Here we report the intraoperative use of optical coherence tomography (OCT) in 3 patients with DCIS. In all 3 cases, additional lesions identified by OCT during surgery were also noted in histopathology reports 3 to 5 days post-surgery, suggesting that intraoperative use of OCT is a valuable tool for margin determination in real-time.
\end{abstract}

Keywords Imaging · Breast cancer · Ductal carcinoma in situ · Invasive ductal carcinoma · Optical coherence tomography

\section{Introduction}

The goal of breast-conserving therapy (BCS) for stage 0 - II breast cancer is to prevent local recurrence by removing diseased tissue completely, while preserving as much healthy tissue as possible for maximal cosmetic and functional outcome [1-3]. Oncologic surgeons typically approach this by excising a volume of tissue slightly larger than that of the target tumor to obtain an outer layer of presumably healthy tissue known as the tumor margin. The surgeon may also take additional margin of tissue shaved from the walls of the cavity created by the tumor [4-6]. If, on postoperative analysis, the pathologist determines the margins to be free of cancerous cells to a depth of $2 \mathrm{~mm}$ or more from the main tumor for DCIS, and no ink on tumor for invasive cancer, then the chances of local disease recurrence are relatively low. If, however, cancerous cells are detected within or at the edge of this margin (i.e., a diagnosis of positive or close margin), the risk of local recurrence is elevated and the patient may need to return to the operating room for additional tissue resection [7-9]. These re-excision procedures, which can

Beth B. DuPree

beth.dupree@nahealth.com

1 Northern Arizona Healthcare, Verde Valley Medical Center, Sedona, AZ, USA occur in 20 to $30 \%$ of cases, are associated with risk of new surgical complications, delayed initiation of adjuvant treatments, worse cosmetic outcomes, higher total cost of treatment, and increased patient anxiety and discomfort.

The challenge is that gold-standard histopathologic assessment typically requires days to weeks to complete. There are currently no highly effective technologies to facilitate direct, intraoperative assessment of the adequacy of margin resection as part of the surgical workflow, representing a significant unmet clinical need.

Optical coherence tomography (OCT) is a non-invasive method of evaluating tissue that exploits variations in the light-scattering properties of different tissues to produce volumetric images to a depth of 1 to $2 \mathrm{~mm}$. OCT can be considered analogous to the use of tissue-specific soundscattering properties for ultrasound imaging, but with markedly greater resolution [10-13]. We have evaluated the intraoperative use of wide-field OCT (WF-OCT) to guide additional margin cavity shaves in BCS.

\section{Patients and Methods}

These cases were from a small, prospective, multi-center pilot study. The protocol was approved by Western Institutional Review Board and our institution's ethics review committee. All patients gave informed consent for procedures 

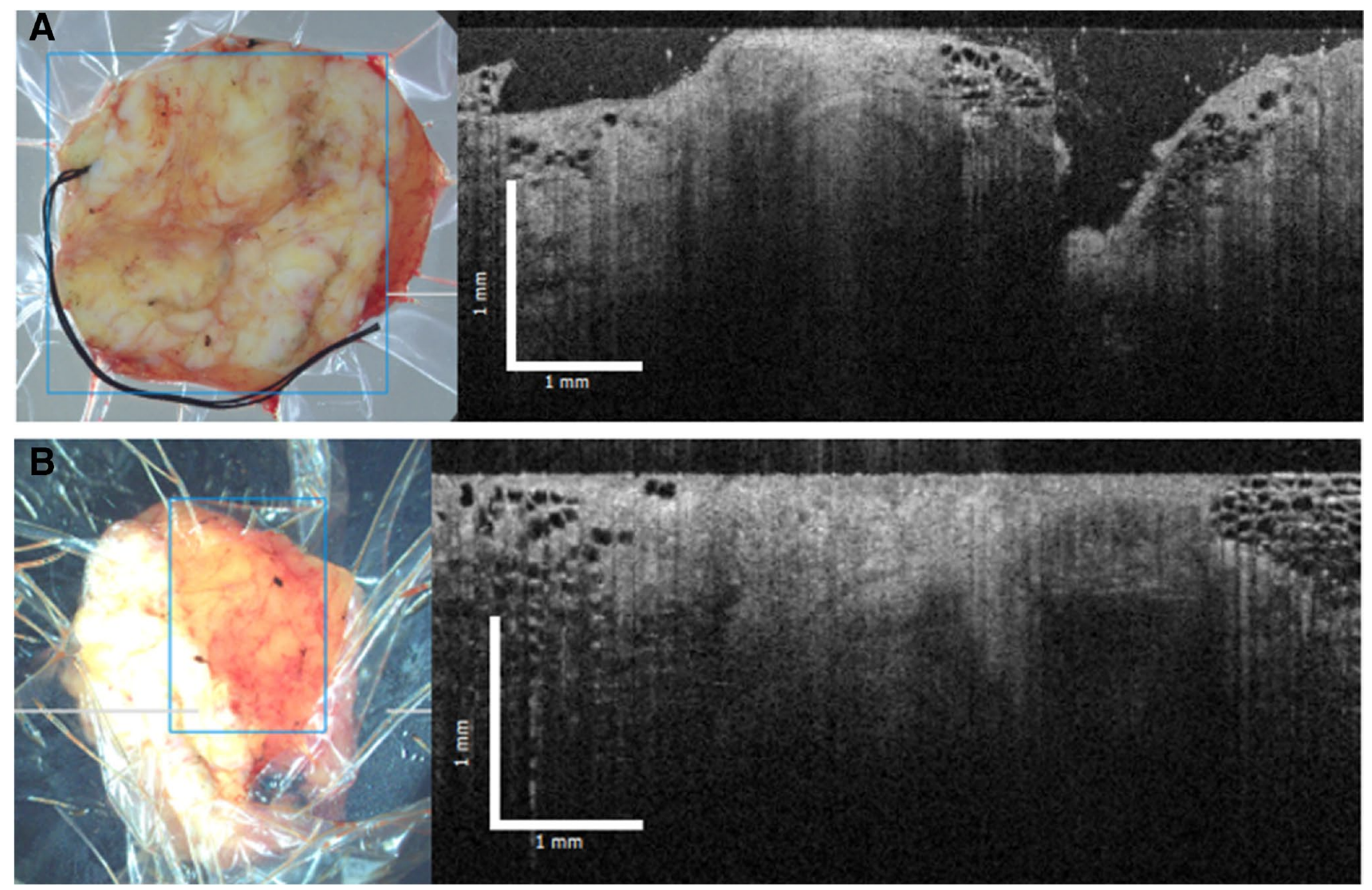

Fig. 1 Patient 1, DCIS-positive posterior and superior lump margins. A, OTIS specimen photograph and WF-OCT scan of the posterior margin showing a suspicious region of interest. B, OTIS specimen

performed. Tumor and cavity shave resections were performed according to standard of care. Fresh lumpectomy specimens were scanned at the time of the surgical procedure using the OTIS ${ }^{\text {TM }}$ 2.0 Optical Coherence Tomography System (Perimeter Medical Imaging AI, Toronto, Canada), according to the manufacturer's operating instructions. Volumes were captured at up to $8.5 \times 8.5 \mathrm{~cm}$ of tissue surface, at approximately $15 \mu \mathrm{m}$ resolution and up to $2 \mathrm{~mm}$ deep. Each margin scanned with OTIS 2.0 was assessed intra-operatively by the trained clinician. Additional margin shaves taken based on OTIS image review and routine tissue assessment of the primary specimen were also imaged and assessed. All specimens were subsequently submitted for standard pathology processing. Overall data analysis was performed at the margin level. Representative regions of interest, marked intra-operatively, are shown in Figs. 1-3.

Patient 1 (a 65-year-old female) underwent a right breast lumpectomy in Jan 2020 for a pre-operative clinical diagnosis of ductal carcinoma in situ (DCIS). Following lumpectomy, all six margins of the lump were scanned by WF-OCT, in the operating room, within the cold ischemic window. The specimen was then processed for comprehensive photograph and WF-OCT scan of the superior margin showing a suspicious region of interest. Scale bars: $1 \mathrm{~mm}$

histopathological evaluation. Time from excision to the pathology report availability was 5 days.

Pathology found a DCIS tumor approximately $2.4 \mathrm{~cm}$ in size, $0.5 \mathrm{~mm}$ from the superior-posterior margin. WF-OCT images also showed suspicious regions of interest on the superior and posterior margins, which were marked intra-operatively. However, as this was a training case, no action was taken to address these margins (Table 1, Fig. 1). The patient returned for a re-excision to remove the additional margins.

Patient 2 (a 76-year-old female) underwent a right breast lumpectomy in Feb 2020 with a pre-op clinical diagnosis of invasive ductal carcinoma (IDC). All specimens and margins were scanned with WF-OCT before histopathology. Time from excision to pathology report availability was 3 days. The deep margin had IDC at $0.3 \mathrm{~mm}$ from the margin, this was also observed on OCT in the intra-operative analysis, however, the margin was the pectoral fascia, and no additional tissue was removed. The actionable posterior margin was already taken down to the pectoral muscle (Table 1 and Fig. 2).

Patient 3 (a 68-year-old female) underwent a left breast lumpectomy in June 2020 for IDC. WF-OCT was used to 
Table 1 Comparison of results from traditional pathology and wide-field optical coherence tomography at the point of care in 3 cases

\begin{tabular}{|c|c|c|c|c|c|}
\hline $\begin{array}{l}\text { Patient/pathology/ } \\
\text { specimen }\end{array}$ & Margin & Pathology & WF-OCT & Cavity shave taken? & $\begin{array}{l}\text { Final } \\
\text { margin } \\
\text { pathology }\end{array}$ \\
\hline \multirow[t]{6}{*}{ Lump } & Posterior & Positive (DCIS 0.5 mm) & Positive & $\mathrm{N}$ & N/A \\
\hline & Anterior & Negative & Negative & $\mathrm{N}$ & N/A \\
\hline & Medial & Negative & Negative & $\mathrm{N}$ & N/A \\
\hline & Lateral & Negative & Negative & $\mathrm{N}$ & N/A \\
\hline & Superior & Positive (DCIS 0.5 mm) & Positive & $\mathrm{N}$ & N/A \\
\hline & Inferior/Lateral & Negative & Negative & $\mathrm{N}$ & N/A \\
\hline \multirow[t]{6}{*}{ Lump } & Lateral & Negative & Negative & N/A & N/A \\
\hline & Medial & Negative & Negative & N/A & N/A \\
\hline & Inferior & Negative & Negative & N/A & N/A \\
\hline & Anterior & Negative & Negative & N/A & N/A \\
\hline & Superior & Negative & Negative & N/A & N/A \\
\hline & $\begin{array}{l}\text { Deep (posterior } \\
\text { pectoral fascia) }\end{array}$ & Negative (IDC 0.3 mm) & $\begin{array}{l}\text { Negative (IDC sus- } \\
\text { picious regions } \\
\text { marked) }\end{array}$ & No (due to pectoral fascia) & N/A \\
\hline \multirow[t]{6}{*}{ Lump } & Posterior & Positive (DCIS 0.6 mm) & Positive & $\mathrm{N}$ & N/A \\
\hline & Medial & Negative & $\begin{array}{l}\text { Negative (IDC sus- } \\
\text { picious regions } \\
\text { marked) }\end{array}$ & $\mathrm{Y}$ & Negative \\
\hline & Anterior & Negative & Negative & $\mathrm{N}$ & N/A \\
\hline & Lateral & Negative & Negative & $\mathrm{N}$ & N/A \\
\hline & Inferior & Negative & Negative & $\mathrm{N}$ & N/A \\
\hline & Superior & Positive (DCIS 0.8 mm) & Positive & $\mathrm{N}$ & N/A \\
\hline
\end{tabular}

"Final margin pathology," pathology of the corresponding shave, if taken, N/A if no shave was taken for that margin. DCIS, ductal carcinoma in situ; IDC, invasive ductal carcinoma

scan all margins of the primary specimen and a medial shave. DCIS was found $0.6 \mathrm{~mm}$ from the posterior margin and $0.8 \mathrm{~mm}$ from the superior margin. These margins were not marked as suspicious during the intra-operative analysis, but regions of interest were found in these scans during a more detailed analysis of the scans by a trained reader. IDC was found on the OCT scan of the medial margin, and action was taken by excising the medial shave. However, the pathology report did not specify the distance of the tumor from the medial margin. Time from excision to the pathology report was 3 days (Table 1 and Fig. 3).

\section{Discussion}

The cavity shave technique has been shown to reduce but not eliminate the incidence of positive margins after BCS [4-6]. Room for improvement remains in margin involvement and with it, the rates of both re-excision and local recurrence [1-7]. An imaging technology that can be used intraoperatively and in real-time to supplement standard-of-care tissue assessment has the potential to improve such outcomes. In this report we have summarized three cases illustrating the utility of WF-OCT, for this purpose.

In a manner analogous to the use of soundwave scattering in ultrasound imaging [10], WF-OCT images are formed based on the interaction between light and the specimen of interest. Microscopic, regional differences in tissue samples possess different optical scattering properties, thus yielding changes in the image density and appearance that correspond to variations in tissue microstructure and composition. Although the penetration depth of OCT is less than that of ultrasound, the use of nearinfrared light yields an image resolution of $15 \mu \mathrm{m}$, which is tenfold greater than that of ultrasound imaging and 100fold greater than that of magnetic resonance imaging. This makes OCT suitable for evaluating subsurface structures in tissue samples obtained through surgical excision.

OCT has been in use for nearly three decades for ophthalmology and intravascular imaging applications [10, 14]; however, until recently the technology was capable of scanning visual fields measuring only a few square millimeters at a time and was therefore impractical for assessment of larger tissue specimens. WF-OCT enables volumetric imaging of $8.5 \times 8.5 \mathrm{~cm}^{2}$ of specimen surface at a depth of up to 


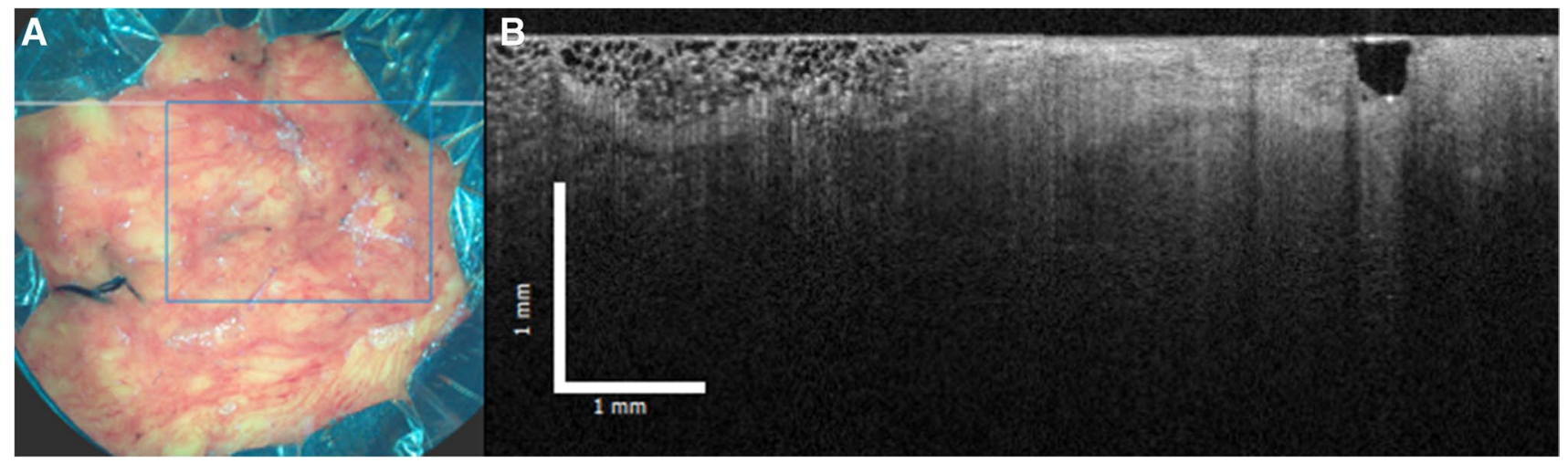

Fig. 2 Patient 2, IDC $0.3 \mathrm{~mm}$ from the posterior margin. A: OTIS specimen photograph and B: WF-OCT scan of the posterior margin showing invasive cancer rising up to the surface of the specimen. Scale bars: $1 \mathrm{~mm}$
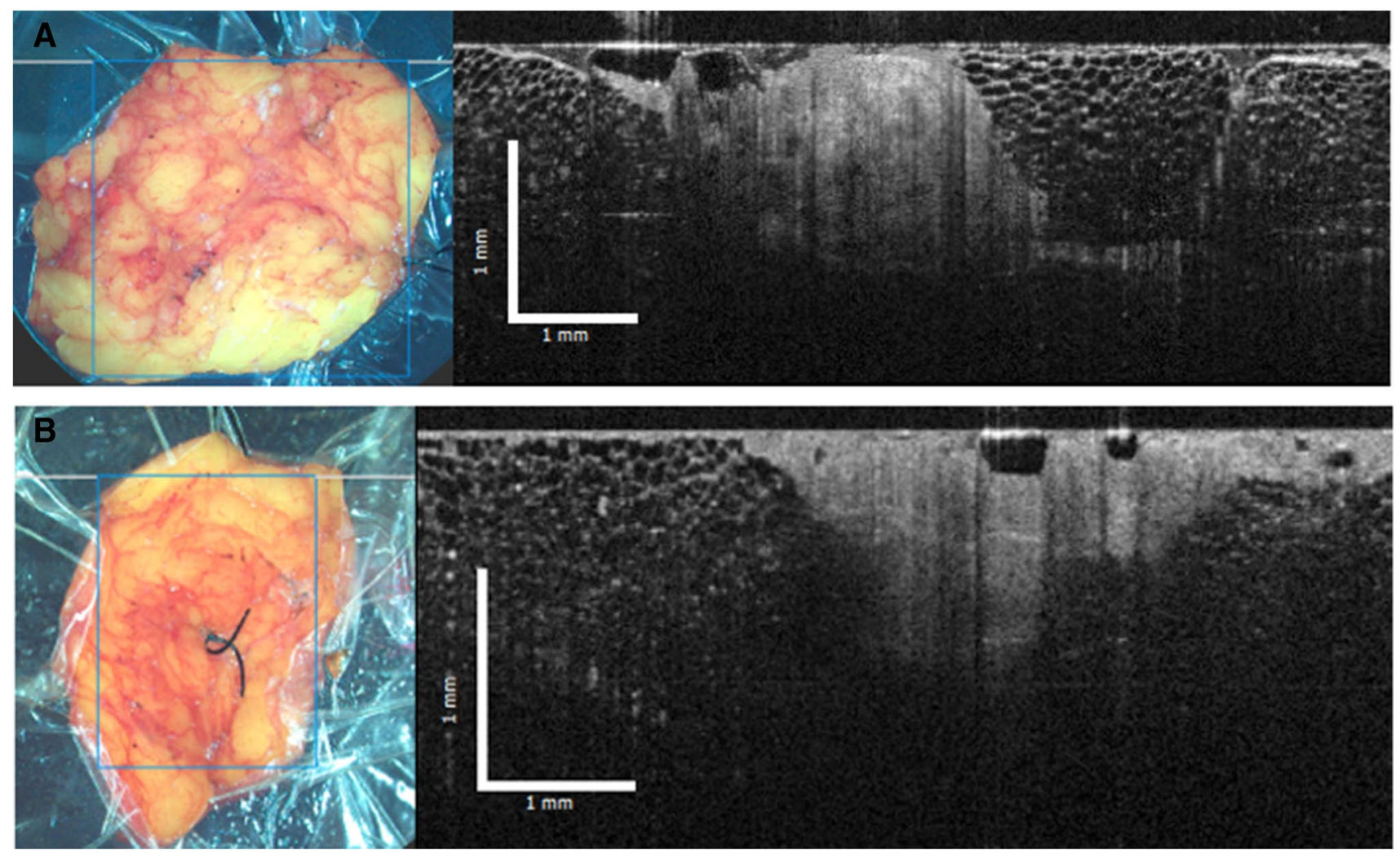

Fig. 3 Patient 3, DCIS-positive $.6 \mathrm{~mm}$ from posterior and $.8 \mathrm{~mm}$ from superior lump margins. A, OTIS specimen photograph and WF-OCT scan of the posterior margin showing a suspicious region

$2 \mathrm{~mm}$, facilitating acquisition of a complete dataset of cross sectional, digital images of the specimen's surface of interest during a single automated scan.

The OTIS 2.0 WF-OCT platform consists of a portable tissue-imaging console and a single-use disposable component that immobilizes the specimen for scanning, in the orientation set by the clinical user. OTIS 2.0 was cleared by the US FDA with an indication for use as an imaging tool of interest. B, OTIS specimen photograph and WF-OCT scan of the superior margin showing a suspicious region of interest. Scale bars: $1 \mathrm{~mm}$

in the evaluation of excised human tissue microstructure by providing two-dimensional, cross-sectional, real-time depth visualization, with image review manipulation software for identifying and annotating regions of interest. The safety and effectiveness of the OTIS device for diagnostic analysis (i.e., differentiating normal versus specific abnormalities) in any tissue microstructure of specified disease has not been evaluated. 
WF-OCT acquires volumetric data, akin to digital, serial cross-sections. With inked specimens, the WF-OCT images can be compared to histopathology sections prepared using the same physical orientation and perspective. As demonstrated in Figs. 1-3, the key, basic features of breast tissue microstructure were readily observable even to the untrained eye.

The results shown in these cases support the findings of a recent reader study by $\mathrm{Ha}$ and colleagues [11]. The readers were trained to distinguish non-suspicious from suspicious findings in WF-OCT images. After a mean of just $3.4 \pm$ $1.2 \mathrm{~h}$ with a training dataset, readers were shown WF-OCT images and asked to identify regions suspicious of cancer while being blinded to other clinical data including histopathology report. Readers showed overall $80 \%$ sensitivity, $87 \%$ specificity, and $87 \%$ accuracy, with breast radiologists and pathologists achieving the highest accuracy.

Thus, with adequate training, pathologists, radiologists, and oncologic surgeons can use the information from WFOCT to supplement gross and microscopic evaluation of breast specimens at the time of excision. If such information were available as part of the surgical workflow, it has the potential to either increase confidence that the tumor has been excised with sufficient margins, or to indicate that additional cavity shavings are advisable prior to closing.

While WF-OCT would not replace a detailed post-operative pathological analysis and confirmatory diagnosis, it could help to reduce the incidence of re-excision surgery, which is highly desirable for clinical outcomes, patient satisfaction, and savings in healthcare resources and costs.

To further explore the potential of WF-OCT, a multicenter, randomized controlled study is being planned to assess the impact of the use of WF-OCT for ex vivo imaging on the re-excision rate in BCS.

\section{Conclusion}

The three cases presented here demonstrate the potential capabilities of WF-OCT technology for producing rapid, real-time, volumetric tissue images on the scale of resected breast cancer tumors. Further development of this technology as a supplemental tool for intraoperative imaging of tumor and cavity-shave margin specimens is warranted.

Acknowledgements The authors thank Jeanne McAdara, $\mathrm{PhD}$, for professional assistance with manuscript preparation, which was funded by Perimeter Medical Imaging AI, Inc. The execution of the referenced clinical studies and WF-OCT image review were supported by Perimeter employees (Maggie Burns), with oversight from the PI for the clinical site. Perimeter also provided the device to conduct the study.

\section{Declarations}

Ethics Approval and Consent to Participate The patients described in this report were participating in a small, unpublished pilot study sponsored by Perimeter Medical Imaging. The protocol was approved by Western Institutional Review Board and our institution's ethics review committee. All patients gave informed consent for procedures performed. The sponsor provided the investigator with the device to conduct the study and a subject fee.

Conflict of Interest The authors declare no competing interests.

Open Access This article is licensed under a Creative Commons Attribution 4.0 International License, which permits use, sharing, adaptation, distribution and reproduction in any medium or format, as long as you give appropriate credit to the original author(s) and the source, provide a link to the Creative Commons licence, and indicate if changes were made. The images or other third party material in this article are included in the article's Creative Commons licence, unless indicated otherwise in a credit line to the material. If material is not included in the article's Creative Commons licence and your intended use is not permitted by statutory regulation or exceeds the permitted use, you will need to obtain permission directly from the copyright holder. To view a copy of this licence, visit http://creativecommons.org/licenses/by/4.0/.

\section{References}

1. Papanikolaou IG, Dimitrakakis C, Zagouri F et al (2019) Paving the way for changing perceptions in breast surgery: a systematic literature review focused on oncological and aesthetic outcomes of oncoplastic surgery for breast cancer. Breast Cancer 26(4):416-427

2. Fajdic J, Djurovic D, Gotovac N, Hrgovic Z (2013) Criteria and procedures for breast conserving surgery. Acta Inform Med 21(1):16-19

3. Rahman GA (2011) Breast conserving therapy: a surgical technique where little can mean more. J Surg Tech Case Rep 3(1):1-4

4. Chagpar AB, Killelea BK, Tsangaris TN et al (2015) A randomized, controlled trial of cavity shave margins in breast cancer. N Engl J Med 373(6):503-510

5. Corsi F, Sorrentino L, Bonzini M et al (2017) Cavity shaving reduces involved margins and reinterventions without increasing costs in breast-conserving surgery: a propensity score-matched study. Ann Surg Oncol 24(6):1516-1524

6. Kobbermann A, Unzeitig A, Xie XJ et al (2011) Impact of routine cavity shave margins on breast cancer re-excision rates. Ann Surg Oncol 18(5):1349-1355

7. Houssami N, Macaskill P, Marinovich ML, Morrow M (2014) The association of surgical margins and local recurrence in women with early-stage invasive breast cancer treated with breast-conserving therapy: a meta-analysis. Ann Surg Oncol 21(3):717-730

8. Kunos C, Latson L, Overmoyer B et al (2006) Breast conservation surgery achieving $>$ or $=2 \mathrm{~mm}$ tumor-free margins results in decreased local-regional recurrence rates. Breast J 12(1):28-36

9. Marinovich ML, Azizi L, Macaskill P et al (2016) The association of surgical margins and local recurrence in women with ductal carcinoma in situ treated with breast-conserving therapy: a metaanalysis. Ann Surg Oncol 23(12):3811-3821 
10. Drexler W, Fujimoto JG (2008) Optical coherence tomography: technology and applications. Springer International Publishing, New York, NY, p 1346

11. Ha R, Friedlander LC, Hibshoosh H et al (2018) Optical coherence tomography: a novel imaging method for post-lumpectomy breast margin assessment-a multi-reader study. Acad Radiol 25(3):279-287

12. Wang J, Xu Y, Boppart SA (2017) Review of optical coherence tomography in oncology. J Biomed Opt 22(12):1-23
13. Vakoc BJ, Fukumura D, Jain RK, Bouma BE (2012) Cancer imaging by optical coherence tomography: preclinical progress and clinical potential. Nat Rev Cancer 12(5):363-368

14. Guagliumi G, Sirbu V (2008) Optical coherence tomography: high resolution intravascular imaging to evaluate vascular healing after coronary stenting. Catheter Cardiovasc Interv 72(2):237-247

Publisher's Note Springer Nature remains neutral with regard to jurisdictional claims in published maps and institutional affiliations. 